\title{
LES ÉQUATIONS AVEC HAMILTONIENS DÉPENDANT DU TEMPS ET LES PHÉNOMĖNES DE RÉSONANCE EN MÉCANIQUE ONDULATOIRE
}

\author{
Par J. WINTER.
}

\begin{abstract}
Sommaire. - Un examen des circonstances expérimentales nous conduit à donner à la relation $\Delta E . \Delta t \geqslant h$, un domaine d'application plus étendu que celui qu'on lui attribue d'habitude et à considérer comme inobservables les phenomènes de résonance.
\end{abstract}

1. Les équations avec Hamiltonien dépendant du temps. - La plupart des problèmes traités dans les ouvrages classiques de mécanique ondulatoire (tels que les déterminations des spectres, les problèmes de diffraction) sont des problèmes stationnaires. L'hamiltonien ne dépend pas du temps et il n'y a pas de difficultés dans la définition expérimentale des valeurs propres de l'énergie. L'on a été naturellement conduit à s'écarter de ce cadre et à aborder des problèmes non stationnaires. Le but poursuivi était double :

$1^{\circ}$ Aborder l'étude d'un ensemble de faits sur lesquels la mécanique ondulatoire doit donner des résultats intéressants, tels que tous les phénomènes d'interaction de la matière et de la lumière, ou encore les phénomènes de perturbation non adiabatiques.

$2^{\circ}$ Justifier les règles de sélection et d'intensité, posées en quelque sorte a priori. Ces règles représentent tout ce que nous pouvons dire sur les transitions dans un système en équilibre isotherme, et il était naturel de chercher à les ramener à des principes généraux.

D'autre part, la forme le l'équation d'onde

$$
H \Psi+h / 2 \pi i \cdot \frac{\partial \Psi}{\partial t}=0
$$

se prêtait facilement à une extension : il suffit d'admettre que $\mathbf{H}$ peut être une fonction donnée du temps. On aura, alors un instrument permettant d'étudier les problèmes d'évolution, en partant de conditions initiales données, à la manière d'un problème de Cauchy habituel ( $\left.{ }^{1}\right)$.

L'on a ainsi traité un ensemble de problèmes et donné des justifications théoriques des règles de sélection et de l'hypothèse adiabatique d'Ehrenfest, et souvent l'on a perdu de vue que le point de départ n'était pas absolument rigoureux. Il serait, à notre avis, très injuste de traiter sur le même pied tous les problèmes où interviennent des Hamiltoniens fonctions du temps: D'abord, considérons ceux qui traitent des interactions radiations électromagnétiques-matière. On n'a jamais eu la prétention de les avoir résolus avec rigueur et, par contre, l'on a obtenu, sur ce terrain, des résultats très intéressants (voir, à ce sujet, notamment le "Wellenmechanischer Ergänzungsband" de Sommerfeld). Nous serions donc mal fondé d'élever des critiques à leur sujet.

Mais il n'en va pas de même pour les problèmes d'évolution non adiabatiques, traités à l'aide de la méthode de variation des constantes. Ce qui caractérise ces problèmes, et les différencie des précédents, c'est l'intervention d'une origine des temps privilégiée, d'un « sharp beginning " pour employer l'expression de Dirac. D'une part, il n'y a pas, à ma connaissance, de justifications expérimentales à donner à l'appui des raisonnements ici employés. D'autre part, ces raisonnements, semble-t-il, prêtent à objection. Mais, par contre, ils sont en accord avec les règles de sélection et d'intensité des raies spectrales, et

(i) Pour un exposé général : Cf. L. de Broglie Introduction à l'étude de la mécanique ondulatotre. ParisHermann 1930 . 
avec l'hypothèse d'Ehrenfest. Cela suffit-il à leur donner force de loi? Cette question mérite d'être discutée, et en présentant ici notre opinion, nous n'avons nullement la prétention d'énoncer des vérités certaines.

2. Les critiques de MM. Born et Jordan. - Dans leur ouvrage bien connu (Elesuentare Quantenmechanik, p. 120-121), MM. Born et Jordan ont formulé les premiers une critique des équations avec Hamiltonien dépendant du temps. Cette critique constitue la base de toute notre étude, qui n'en est, en quelque sorte, que le commentaire et la suite. Exposons-la sommairement :

Il n'y a, a priori, aucune raison de traiter la règle d'indétermination concernant les mesures d'énergie et de temps, sur un pied différent de celui des autres règles d'indétermination. Or, l'écriture $\boldsymbol{H}(\boldsymbol{t})$ où $\boldsymbol{H}$ est le hamiltonien d'un système, implique une connaissance simultanée de ce hamiltonien et du temps. D'où contradiction. Est-il possible de lever cette contradiction?

MM. Born et Jordan proposent de dire ceci : Toutes les fois qu'on pourra séparer les objets considérés en deux systèmes I et II, tels qu'on puisse négliger l'action de II sur I, mais non celle de I sur II (exemple : I, champ électromagnétique; II, atome, quand l'on peut négliger l'énergie rayonnée par rapport à celle du champ), on pourra dans l'étude. de II, introduire l'écriture $H(t): H$ est le hamiltonien de II, $t$ est le “ temps de l " dont la connaissance n'est pas incompatible avec celle de l'énergie de II. Cette hypothèse, en quelqne sorte relativiste, puisqu'elle introduit deux temps, s'applique-t-elle à tous les problèmes traités avec Hamiltonien contenant le temps? C'est la première question qui se pose.

Il nous semble qu'on peut répondre « oui » dans tous les cas traités usuellement, sauf dans le problème dit “ de la résonance de deux systèmes couplés 》 (Elementare Quantenmechanik, §65; Heisenberg Die physikalischen prinzipien der Quantentheorie, partie mathématique par. 5).

Cette restriction proposée par MM. Born et Jordan, est-elle suffisante pour rendre les raisonnements rigoureux? Ici, nous devons bien avouer que nous ne le pensons pas. Le temps propre d'un système microscopique est, bien entendu, quelque chose d'inobservable directement; il faudrait que la suite des calculs fasse jouer à ces deux temps des rôles bien distincts et cela ne nous semble pas réalisé : pour établir les équations, dites de « variation des constantes » (1) on porte l'expression :

$$
\Psi=\sum_{n} C_{n}(t) \Psi_{n}
$$

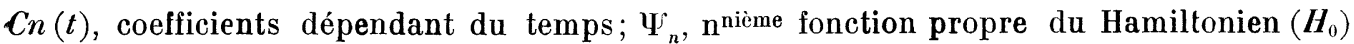
dans l'équation

$$
\left[\boldsymbol{H}_{0}+H_{1}(t)\right] \Psi+\frac{h}{2 \pi i} \frac{\partial \Psi}{\partial t}=0\left(^{2}\right) .
$$

La dérivée partielle est prise par rapport au temps du système II et on lui fait opérer des $\mathrm{C}$ qui sont fonctions du temps du système I. A eette objection purement formelle, viendra s'ajouter une objection expérimentale que nous développerons plus loin : comment peut on repérer l'instant initial d'une interaction?

Nous croyons donc qu'ici, la seule méthode de discussion possible est celle qui a été si souvent adoptée par MM. Bohr et Heisenberg: Chercher quel est le sens expérimental des expressions employées, et notamment de celle-ci : Un système dont le Hamiltonien est connu, subit à l'instant $t$ une perturbation qui se traduira par une modification donnée de cet hamiltonien.

A cet effet, nous utiliserons une étude due à M. Schrödinger [Zeitschrift für physik, t. 78 (1932), p. 309].

(1) Pour la comparaison des cas où la perturbation provient d'un système quantifié ou non quantifié, nous renvoyons à une étude de M. L. Brillouin : Notions de mécanique ondulatoire. Les méthodes d'approximation. Paris-Hermann et Cie 1932.

(2) $H_{0}$ Hamiltonien constant. $H_{1}(t)$ terme perturbateur qui s'annule pour $t=0$. 
3. L'Etude de M. Schrödinger. - MM. Rausch von Traubenberg et Gebauer ont cherché à mettre en évidence une perturbation non adiabatique :

Pour cela, ils ont dirigé un courant de rayons canaux (atomes d'hydrogène) dans deux entrefers (fig. 1) où des champs opposés, tous deux perpendiculaires au sens du courant, étaient proluits par des plaques de condensateurs. On projette, à l'aide d'un appareil optique, l'image du courant d'atomes sur la fente d'un spectroscope, et on observe les raies ainsi obtenues : On obtient un entrecroisement, dû au changement brusque de l'effet Stark, en 0 (centre de la figure). La figure 2 schématise grossièrement les raies ainsi photographiées Au croisement des raies a-t on le droit de parler de l'observation d'une perturbation non adiabatique?

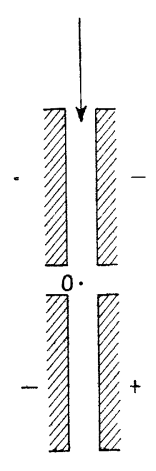

Fig. 1.

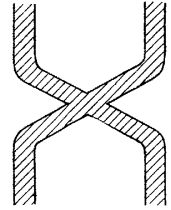

Fig. 2.

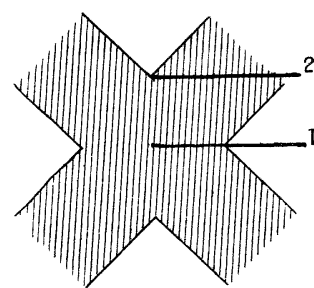

Fig. 3.

Cette question fait l'objet de l'étude de M. Schrödinger : il suppose l'atome considéré fixe et soumis à une perturbation donnée en fonction du temps $\boldsymbol{F}(t)$ (l'expression de $\boldsymbol{F}$ résultera de la vilesse des rayons canaux et de la forme du champ). Il applique les équations de variation des constantes, et fait intervenir la durée moyenne 0 pendant laquelle l'atome observé traversera le disque de diffraction correspondant à l'appareil optique utilisé et à un point de la plaque photographique. Pour avoir les termes spectraux, il faudra développer en série de Fourier l'expression des fonctions d'onde, multipliées par un facteur, fonction du temps, qui représentera la durée de passage de l'atome à travers le disque de diffraction : On aura ainsi la valeur des termes spectraux au point de la plaque pholographique correspondant au disque de diffraction considéré (ou à l'intervalle de temps, puisque dans le calcul l'atome est supposé fixe).

Ceci posé, est-il possible d'observer une déformaiion des raies qui se soit produite pendant une durée inférieure à la période propre de l'effet Stark qui lui a donné naissance? C'est la définition mème du non adiabatisme.

La conclusion est la suivante : Pour un pouvoir séparateur optimum de l'appareil optique utilisé, l'on pourra rendre $\theta$ (durée de parcours du disque de diffraction) égal à $t$, (temps au bout duquel la période propre de l'effet Stark est précisément à $t$. si l'on suppose que cet effet est nul au temps o), et la largeur des raies sera alors égale au déplacement provoqué par l'effet Stark. Si les raies forment une sorte de $\mathbf{X}$, l'observation du phénomène non adiabatique consistera à observer une déformation des raies entre les niveaux 1 et 2 (fig. 3 ) ( 1 , centre de la figure, $y$, point où les branches de l'X bifurquent).

Si la condition de pouvoir séparateur optimum n'est pas réalisée, le chevauchement des raies empêchera de discerner leur séparation sur une durée plus courte que la période propre de l'effet Stark correspondant.

Etudions de plus près cet important résultal : 
Distinguons pour cela les cas suivants :

a) Les atomes ne rayonnent pas et sont dans un état non dégénéré.

Le problème n'a pas d'applications intéressantes.

b) Les atomes rayonnent et oscillent entre deux états non dégénérés.

On retrouve la règle d'indétermination bien connue $\Delta E \times \Delta l \supseteq h ; \Delta t$ est ici $\theta$, indétermination sur l'instant d'émission d'un photon, c'est-à-dire sur l'instant d'une transition.

$\Delta E$ est donné par la largeur de la raie; c'est l'indétermination sur l'énergie mise en jeu par la transition. Ce résultat est une simple conséquence de la diffraction.

Pour préciser la signification de l'écriture $\boldsymbol{H}(\boldsymbol{t})$, on a la restriction suivante : Une variation de $\boldsymbol{H}$ ne peut être fixée dans un intervalle de temps inférieur à la période propre définie par les différences des variations imposées aux deux niveaux. Ceri est encore une forme du principe d'indétermination : l'atome (') acquiert au passage de la discontinuité une énergie qui sera soit $\left({ }^{2}\right)\left(E_{1}^{\prime}-E_{1}\right)$, soit $\left(E_{2}^{\prime \prime}-E_{2}\right)$ selon l'état où il se trouve (fig. 4). L'indétermination sur le gain d'énergie est donc $\left(E_{1}^{\prime}-E_{1}\right)-\left(E_{2}^{\prime}-E_{2}\right)=\Delta E$; l'indétermination sur l'instant du passage de la discontinuité doit donc être $\frac{h}{\Delta E}$, c'est le résultat de Schrödinger, qui pouvait donc être annoncé, à priori, sans calcul.

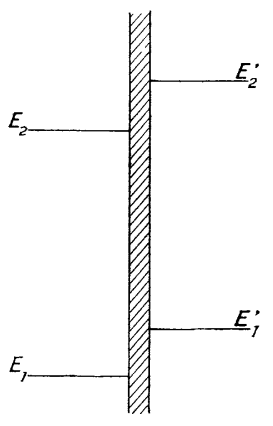

Fig. 4.

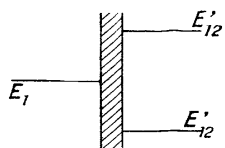

Fig :.j.

c) Les atomes ne rayonnent pas, mais sont dans un état dégénéré avant la discontinuité de champ; celle ci lève la dégénérescence.

Le résultat de M Schrödinger s'applique encore avec quelques petites modifications de calcul sans importance, M. Schrödinger a supposé la fonction d'onde initiale adaptée à la perturbation, c'est à-dire que le niveau ne se scinde pas. Le problème n'aurait alors pas d'intérêt. Si le niveau se scinde, et s'il n'y a pas rayonnement, les $\nu_{k l}$ de M. Schrödinger n'interviennent pas. Mais on aura des $\omega_{k 1},{ }_{k 2}$, fréquences propres d'effet Stark entre états primitivement confondus et séparés par la perturbation On obtiendra un résultat analogue : l'instant où se produit la perturbation sur un atome donné doit être considéré comme indéterminé à $\theta$ près ( $\theta$ période propre due à la perturbation) c'est-à-dire $h / \boldsymbol{E}_{12}^{\prime \prime}-{E^{\prime}}_{11}$ (fig. 5).

4. Application aux phénomènes de résonance. - Remarquons que les circonstances ci-dessus étudiées, en $\mathrm{C}$, sont précisément celles d'une expérience qui permettrait de mettre en évidence un phénomène de résonance $\left(^{3}\right)$. Le courant d'atomes dans un

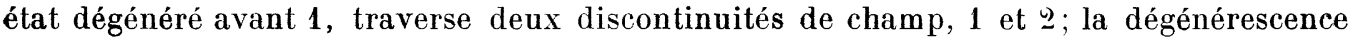

(1) Nous admettons, ici, que latome qui rayonne se trouve tantòt dans l'état $E_{1}$, tantôt dans l'état $E_{2}$.

${ }^{(2)}$ Les accents indiquent les niveaux perturbés : $E_{1}$ devient $E_{1}^{\prime}$.

${ }^{(3)}$ Ce phénomène peut être schématisé ainsi : les fonctions propres dégénérées d'un système sont $f_{1} \mathrm{e}^{-\frac{2 \pi i}{h} E_{1} t}$ et $f_{2} \mathrm{e}^{-\frac{2 \pi i}{h} E_{1} t}, f_{1}$ et $f_{2}$ caractérisés par les valeurs d'une certaine grandeur $A$, commutable 
est levée en 1 et rétablie en 2 (fig. 6). Selon les variations de la distance $D$, on observerait audelà de 2 des proportions variables d'atomes dans les divers états dégénérés. Cette conclusion de la théorie de la résonance est-elle acceptable? Non, si nous nous en tenons aux résultats de l'étude précédente; car les instants où les perturbations 1 et 2 se produisent sur un atome seraient indéterminés à une période propre de résonance près.

Reprenons donc l'examen du phénomène de résonance en supposant acquises les règles énoncées ci-dessus : nous parlerons simplement de la résonance intérieure : un système se trouve dans un état d'énergie dégénéré ; une perturbation extérieure lève la dégénérescence et scinde le niveau initial : le système oscillera alors entre les divers états dégénérés primitifs, et la période d'oscillation sera donnée par l'expression $\left(\boldsymbol{h} / \boldsymbol{E}^{\prime \prime}{ }_{12}-\boldsymbol{E}_{11}^{\prime}\right)$ (fig. 5) $\boldsymbol{E}_{12}^{\prime} . \boldsymbol{E}_{11}^{\prime}$ désignant les niveaux scindés, que nous supposerons au nombre de deux.

Pour observer l'oscillation du système qui est en étal d'énergie statistiquement indéterminée, il faut lever brusquement la perturbation et procéder ensuite sur le système à une mesure de la grandeur $A$

Fig. 6. qui distingue les états dégénérés (voir note ci-dessus); par exemple si $A$ est la valeur du nombre quantique $m$ pour des atomes $P$, il faudra faire une expérience de Stern-Gerlach. La cessation de la perturbation est une circonstance essentielle, sans laquelle la notion d'oscillation entro les états dégénérés primitifs n'aurait pas de sens. Il est également essentiel de supposer le système perturbé dans un état d'énergie indéterminée, c'est-à-dire dans un état représenté par une combinaison des états perturbés purs, correspondant aux valeurs propres $\boldsymbol{E}_{12}^{\prime \prime}, \boldsymbol{E}^{\prime \prime}{ }_{11}$.

Mais s'il est impossible de donner un sens expérimental à l'instant de cessation de la perturbation, si ce n'est à une période propre de résonance près, il est évident que le phénomène s'évanouit.

Supposons que nous cherchions à déterminer cet instant avec une plus grande précision : l'indétermination sur l'énergie mise en jeu au cours de la cessation de la perturbation croîtra, de façon qu'on ait $\Delta E \times \Delta t \supseteq h$. ll faudra dans l'expression de la fonction d'onde de l'état perturbé, faire intervenir des termes autres que ceux en

$$
\mathbf{e}^{\frac{2 \pi i}{h} \cdot E^{\prime}{ }^{2} t} \text { et } \mathbf{e}^{\frac{2 \pi i}{h} E_{11}^{\prime} \cdot t} .
$$

Ces termes se déphaseront par rapport aux précédents avec des vitesses modifiées, et un calcul simple permet de voir que l'influence de ces termes fera disparaitre la prédominance à intervalles fixes du facteur de $f_{1}$ sur celui de $f_{2}$ et inversement dans l'expression de la fonction d'onde complète.

Cette critique peut être faite d'une façon analogue, nous semble-t-il, à certains problèmes traités à l'aide de la méthode de variation des constantes; nous nous contenterons d'écarter ici une objection :

Nous n'avons pas fait intervenir l'ensemble des niveaux énergétiques du système

avec l'énergie. Après perturbation, elles deviennent $F_{1} \mathrm{e}^{-\frac{2 \tau i}{h} E_{11}^{\prime} t}$ et $F_{2} \mathrm{e}^{-\frac{2 \pi i}{h} \cdot E_{12}^{\prime} t,}$ avec $F_{1}=f_{1}+f_{2}$ et $F_{2}=t_{1}-f_{2}$. Si l'atome est dans l'état $f_{1}$ avant la perturbation, après la perturbation il sera dans l'étal $F_{1} \mathrm{e}^{-\frac{2 \pi i}{h} \cdot E^{\prime}{ }_{11} t}+F_{2} \mathrm{e}^{-\frac{2 \pi i}{h} E_{12}^{\prime} t} ;$ ces termes se déphaseront et viendront en opposilion. Si à ce moment la perturbation cesse, la fonction d'onde sera $F_{1}-F_{2}=f_{2}$; on retrouvera l'atome dans l'état $f_{2}$, c'ost-à-dire avec une valeur différente de la grandeur $A$. (Pour une étude complète, Cf. Elementare quantenmechanik, Die Physikalischen Prinzipien der quantentheorie.)

(1) On aura, par exemple, une expression telle que :

$$
F_{1}\left\{\mathrm{e}^{-\frac{2 \pi i}{h} E_{11}^{\prime} t}+\mathrm{e}^{-\frac{2 \pi i}{h}\left(E^{\prime}{ }_{11}+\frac{\Delta E}{2}\right)}\right\}+F_{2}\left\{\mathrm{e}^{-\frac{2 \pi i}{h} E_{12}^{\prime} t}+\mathrm{e}^{-\frac{2 \pi i}{h}\left(E_{12}^{\prime}-\frac{\Delta E}{2}\right) \iota}\right\}
$$


après perturbation; en toute rigueur, il faut développer la fonction d'onde originale, en série des fonctions propres perturbées : toutes ces fonctions propres doivent intervenir. Mais il est facile de voir qu'on ne peut pas ainsi retrouver la règle $\Delta E \times \Delta t \supseteqq h$.

Il suffit de supposer une perturbation discontinue et instantanée, alors $t=0$. On obtiendra $\Delta \boldsymbol{E}$ en développant la fonction d'onde primitive, qn'on peut supposer être une ancienne fonction propre par rapport aux fonctions perturbées. La valeur moyenne de $\boldsymbol{E}$ sera obtenue en faisant la moyenne des nouvelles valeurs /propres, affectées du coefficient de probabilité des fonctions propres correspondantes, et $\Delta E$ sera (cf. Heisenberg Die Physikalischen Prinzipien der Quantentheorie, p. 11), l'erreur quadratique moyenne définie d'une façon analogue. Il faudrait que $\Delta E$ soit toujours infini. Un exemple simple montrera qu'il n'en est rien.

Il suffit de développer en série de Fourier, une des fonctions propres de l'oscillateur harmonique linéaire (ceci correspond à la perturbation qui consisterait à supprimer brusquement le centre attractif ; les nouvelles fonctions propres sont l'ensemble des fonctions $\mathrm{e}^{i k x}, k$ prenant toutes les valeurs de $-\infty$ à $\left.+\infty\right)$ pour se convaincre qủe $\Delta E$ est bien fini.

5. Peut-il y avoir discontinuité d'énergie sans déphasage? - L'on peut admettre (cf. Heisenberg Die Physikalischen Prinzipien der Quantentheorie, page 40̃) que les deux circonstances suivantes sont essentiellement différentes.

a) Une perturbation est effectuée, mais on ne procède à aucune mesure d'énergie (ni rayonnement, ni expériences de Stern-Gerlach alors la phase reste continue pendant la perturbation).

b) On procède, à la suite de la perturbation, à des mesures d'énergie: la phase devient alors indéterminée.

Si l'on adopte ce point de vue, toute notre discussion devient sans valeur. Mais la question essentielle est de savoir si cette distinction a un sens physique cerlain : La règle $\Delta E . \Delta \ell \leqslant h$ ne vaudrait qu'au cours d'une mesure effective de l'énergie; mais une perturbation qui nous permettrait cependant d'affirmer qu'à un instant $t$ une particule a reçu une quantité d'énergie $E$, ne constituerait pas une mesure d'énergie. La distinction nous semble très abstraite :

Si on dirige un courant de corpuscules à travers une ouverture diaphragmée, nous effectuons une mesure des coordonnées par cela même, sans qu'il soit besoin de regarder ce qui se passe à l'aide de photons. Le diaphragme perturbera les coordonnées de moments de lui-même; ce phénomène constitue la diffraction.

La question qui se pose, est de savoir si une perturbation brusque de l'énergie, n'entrainera (ipso facto » une perturbation de la phase, par un phénomène analogue à celui de la diffraction. Le déphasage se produirait alors sans “mesure de l'énergie. C'est le point de vue que nous avons implicitement adopté ici. Il nous semble plus conforme à l'esprit de la nouvelle mécanique.

Pour conclure, nous dirons qu'il nous parait difficile de donner aujourd'hui un formalisme satisfaisant pour étudier l'influence d'une variation rapide de l'Hamiltonien. Ce formalisme, pour ne pas s'écarter de l'expérience, devrait traduire la règle $\Delta E \times \Delta t \gg h$; or, pour cela, il suffirait d'admettre que toute modification de l'énergfe d'un système se fasse par émission ou absorption de radiations; ceci nous ramène au problème de l'interaction de la matière et des ondes électromagnétiques, non entièrement résolu.

Remarque. - Dans toute cette étude, nous n'avons pas parló des noyaux des atomes du courant : nous avons supposé leur vitesse constante sur tout le parcours. Nous aurions pu la supposer variable: cela nous aurait permis d'exposer la question d'une manière différente, mais le résultat de la discussion serait le même.

Nous prierons, en terminant, Messieurs L. de Broglie et L. Brillouin de bien vouloir trouver ici l'expression de ma plus vive reconnaissance pour leurs précieuses critiques et leur grande bienveillance. 